\title{
Entrelacs
}

Cinéma et audiovisuel

Hors-série $n^{\circ} 4$ | 2016

Paysages en séries

\section{Gomorra, la série : la réinvention de l'imaginaire napolitain}

Alfonso Pinto

\section{(2) OpenEdition}

Journals

\section{Édition électronique}

URL : http://journals.openedition.org/entrelacs/2151

DOI : 10.4000/entrelacs.2151

ISBN : $2261-5482$

ISSN : 2261-5482

Éditeur

Éditions Téraèdre

Édition imprimée

Date de publication : 1 novembre 2016

ISSN : 1266-7188

\section{Référence électronique}

Alfonso Pinto, «Gomorra, la série : la réinvention de l'imaginaire napolitain », Entrelacs [En ligne], Horssérie $n^{\circ} 4$ | 2016, mis en ligne le 06 février 2018, consulté le 19 avril 2019. URL : http:// journals.openedition.org/entrelacs/2151; DOI : 10.4000/entrelacs.2151

Ce document a été généré automatiquement le 19 avril 2019

Tous droits réservés 


\title{
Gomorra, la série : la réinvention de l'imaginaire napolitain
}

\author{
Alfonso Pinto
}

Grâce à un statut épistémologique assez fluide, les études géographiques ont commencé depuis quelques années à s'intéresser aux représentations spatiales offertes par différents supports hétérogènes. En particulier, le cinéma a suscité l'intérêt des géographes à partir de la fin des années 1970. Quelques contributions sporadiques, quelques analyses ponctuelles, ont cédé la place à partir des années 1990 à des travaux plus complets, tant sur un plan théorique que sur celui de certaines phénoménologies spatialement pertinentes. Le chemin est encore long, mais l'actualité récente est encourageante. Fait révélateur, une revue comme les Annales de Géographie dédie un numéro spécial (2014) aux rapports entre le septième art et les études sur les espaces. La géographie a, depuis son apparition, montré un intérêt particulier pour les images, au point que certains ont jugé que cette discipline était essentiellement axée sur les représentations ${ }^{1}$. On pense à la carte, bien évidemment, mais on peut, prudemment, en élargir l'éventail.

Le but de cette contribution est de réfléchir, d'un point de vue géographique, au rôle que le film de Matteo Garrone, Gomorra (2008), et la série homonyme de 2014 ont joué dans la réinvention de l'imaginaire spatial napolitain.

En revanche, avant de pouvoir cibler l'attention sur ces deux produits audiovisuels, il faudra remarquer la géographicité de cette approche, notamment en précisant brièvement ce que l'on entend ici par « imaginaire de l'espace ». En effet, le géographe peut se rapporter à un produit audiovisuel selon deux postures principales : la première, axée sur le concept de représentation, l'autre, au contraire, sur les pratiques de fabrication des imaginaires ou, si l'on veut, sur la constitution de ce que l'on peut définir comme un « régime de visibilité ».

Dans le détail, notre analyse débutera avec un regard sur le film de Garrone, qui, en s'inscrivant pleinement au sein de la longue et prolifique tradition néoréaliste italienne, s'efforce d'offrir un regard le plus naturel possible sur les réalités socio-spatiales de la banlieue napolitaine et en particulier sur le rôle néfaste du phénomène criminel de la camorra. Il s'agit d'une approche somme toute logique, en plein accord avec les intentions 
du film qui, on le sait, met en image certains épisodes décrits dans le roman-enquête homonyme du journaliste Roberto Saviano (2006).

5 La série, produite par la chaîne privée Sky Italia à partir du 2014 et composée désormais de deux saisons (la deuxième sortie en mai 2016), présente un univers entièrement fictionnel, bien que vraisemblable. En revanche la véritable innovation concerne le mélange de différents codes esthétiques qui suggèrent une analyse axée sur l'imaginaire. Tout en gardant certains traits néoréalistes, les réalisateurs (Stefano Sollima, Francesca Comencini, Claudio Cupellini) ont cherché à fabriquer un univers diégétique totalement inédit, tant pour la ville de Naples que pour l'Italie en général. Il s'agit d'un imaginaire qui repose notamment sur une sorte de métropolisation de l'espace, et qui se réfère aux grandes fictions urbaines nord-américaines (séries ou même long-métrages).

6 Le résultat est donc celui d'un univers hétérodoxe, inédit, capable d'enrichir, sans pour autant effacer, l'imaginaire varié et multiforme de la ville parthénopéenne.

\section{Approches géographiques : représentation et imaginaire}

7 Nombreuses sont les difficultés que les géographes ont dû affronter dans leurs rapports à l'image en mouvement et en particulier aux films. Premièrement, le cinéma est un objet trompeur. Sa profonde adhérence mimétique à la réalité a été, bien à raison, considérée comme une forte contrainte. C'est par exemple le sens de l'analyse effectuée par Michel Foucher sur le cinéma western, en 1976, travail précurseur dans ce domaine. Selon le géographe, les films appartenant à ce genre reposent sur un profond décalage entre lieux de tournage et lieux historiques. Souvent, les événements au cœur des narrations cinématographiques se sont effectivement déroulés dans un contexte qui n'est pas du tout celui du désert et des grands canyons. Mais Foucher indique qu'il y a bien plus à la tromperie du cinéma que ces simples exigences cinématographiques. Comme la carte, le film est une puissante représentation, mais sa finalité première n'est justement pas géographique. La cartographie elle-même, bien qu'instrument crucial du géographe, n'est pas dégagée de toutes contraintes, au point que nous disposons aujourd'hui d'un recul suffisant pour démentir l'objectivité qui lui a été attribuée pendant plusieurs décennies. Tout comme les données cartographiques, le cinéma est subjectif et partiel. Il repose en permanence sur des questions de point de vue, de décision subjective sur la pertinence des éléments à disposition. Si la carte se concentre sur des données souvent quantifiables, le cinéma, de son côté, grâce à sa capacité de reproduction du paysage de manière non statique, offre des éléments fonctionnels à une approche qualitative.

C'est bien le sens que l'on attribue à une posture représentationnelle, axée sur les décalages et/ou les ressemblances entre les univers diégétiques et extra-diégétiques. On est donc au sein de l'ontologie réaliste, s'exprimant notamment dans la fameuse formule de Bazin (1981) : le cinéma comme «fenêtre sur le monde ».

9 En revanche, le géographe peut « jouir » d'une autre vision sur la question : celle qui vise à réfléchir sur l'imaginaire des lieux, non en tant que représentation, mais en tant qu'espace à proprement parler. On peut définir cet imaginaire comme un intertexte composé de différentes représentations spatiales (dont le cinéma), qui constitue ce que le cinéaste André Gardies (1993) considérait être le savoir préalable du spectateur. Ce patrimoine intersubjectif est, selon lui, à la base de la construction diégétique du récit 
filmique. La diégèse n'est pas une donnée fournie directement par le réalisateur. Elle résulte d'un processus qui implique la participation active (mais toutefois inconsciente) du spectateur. Dans le noir de la salle, ce dernier se trouve face à une complexe série de signes figurés et organisés qui assument leur pleine signification uniquement au travers d'un processus interprétatif. Ce processus dépend d'une démarche dialogique, c'est-à-dire qu'il prend forme à travers l'interaction entre les informations offertes par le film et le savoir préalable du spectateur. L'intervention d'un certain degré de subjectivité est donc indéniable, mais n'est qu'une face de la médaille, dont l'autre est constituée par des variations inscrites au sein d'un patrimoine culturel commun. D'un côté, donc, intervient l'expérience subjective, de l'autre, le savoir préalable, considéré comme un élément intersubjectif.

La géographie, en ce sens, peut - et doit - être sensible à ce processus, et cela en raison d'une considération cruciale pour le statut même de la discipline : le caractère composite de son véritable objet de recherche, c'est-à-dire l'espace humain. Le géographe Michel Lussault a longuement réfléchi sur la nature de ce dernier (2007). En reprenant la dichotomie idéel/matériel postulée par Maurice Godelier en 1992 - reposant sur le constat que le réel est plus grand que la matière - le géographe affirme que «l'idéalité n'est pas l'instance des idées abstraites, mais la pensée, sous toutes ses formes (y compris celles qui la fixent dans des énoncés matériels comme les cartes, les peintures, les objets ou dans des dispositifs-formes comme des bâtiments, des paysages, etc.), en acte(s) dans la construction et la stabilisation des agencements sociétaux et des pratiques des êtres humains » (2007, pp. 69-70). De ce point de vue, l'espace humain serait donc un hybride ou, mieux encore, un composite des deux dimensions, celle matérielle et celle idéelle. Le but de Lussault est donc d'élargir la posture représentationnelle et de théoriser que toute représentation de l'espace rentre au sein de cette hybridité (ou de cette composition) en devenant de l'espace-en-propre. "Cet espace «figuré » par l'iconographie n'est donc pas "seulement » une image spatiale, un double mimétique, une réplique en réduction; c'est aussi et surtout de l'espace-en-propre configuré par l'image » (ibid., p. 73).

11 En revenant donc au processus de construction diégétique décrit par Gardies, on devrait donc mieux saisir la géographicité du fonctionnement filmique, qui, on l'a vu, repose sur un savoir préalable intersubjectif. On peut donc considérer l'imaginaire spatial comme la résultante des différents supports constituant ce savoir.

12 Selon cette perspective, le film acquiert un double statut par rapport à l'imaginaire : d'un côté sa réalité diégétique se construit grâce à l'existence d'un imaginaire dont le spectateur est déjà en possession; de l'autre, une fois la réception achevée, le même film contribue à la fabrication de cet imaginaire, en devenant une nouvelle composante des savoirs des spectateurs.

13 Ce qui se met en place est donc un complexe jeu d'interactions multiples intervenant culturellement au sein de nos relations à l'espace, et qui, d'une certaine manière, aboutit à la constitution de ce que l'on appelle "régimes de visibilité » des lieux, considérés comme l'ensemble des traits récurrents que l'imaginaire spatial d'un lieu précis donne à voir.

14 Bien évidemment, la réflexion que l'on a essayé de synthétiser ici est bien plus riche et complexe et creuse au sein de l'épistémologie-même des disciplines géographiques. Toutefois elle devrait être suffisante pour mieux saisir les enjeux de notre analyse comparée du film de Matteo Garrone et de la série homonyme. Comme on le verra, c'est bien la coprésence de ces deux approches (cinéma comme représentation et cinéma 
comme fabriquant d'imaginaire) qui fonde la pertinence de notre hypothèse, c'est-à-dire la réinvention de l'imaginaire d'une ville.

\section{Gomorra. Le film.}

Genre, filon, courant ou tout simplement manière de concevoir le cinéma, le néoréalisme a sensiblement marqué toute la production cinématographique italienne depuis l'aprèsguerre. À partir des films fondateurs de Rossellini, De Sica ou Fellini, en passant par le " cinéma-civil ${ }^{2}$ ", la prolifération d'œuvre à forte connotation sociale et civique est constante. Acteurs souvent non-professionnels, tournages en extérieur, mise en scène et montage visant à offrir un développement narratif le plus naturel possible en sont les éléments récurrents.

Le film de Matteo Garrone, Gomorra (2008), tiré du roman-enquête du journaliste Roberto Saviano, nous livre un portrait entre la fiction et le documentaire sur le fonctionnement socio-spatial de l'univers de la camorra. La tradition cinématographique des mafias n'est certainement pas une nouveauté, en Italie ou ailleurs. Cependant Garrone offre une manière de filmer qui bouleverse les esprits à cause du profond hyper-réalisme et de l'absence de tout jugement moral ou éthique. Le but est, d'une certaine manière, proche d'un souci sociologique: montrer pour comprendre, en laissant tout jugement au spectateur.

De manière fonctionnelle, les codes néoréalistes sont pleinement respectés. La plupart des acteurs sont des non-professionnels, à l'exception de Toni Servillo et Maria Nazionale (certains d'entre eux ont été jugés ensuite pour liaisons avec la Camorra); la langue employée est un patois napolitain si spécifique que même la version italienne du film a dû être sous-titrée. Enfin - élément ici fondamental - le réalisateur a porté une grande attention à la mise en scène des espaces. Le film se concentre sur les plus fameuses cités de la banlieue napolitaine, comme par exemple Secondigliano ou Scampia (avec ses fameux HLM connus sous le nom de «Voiles »). Plusieurs séquences attirent l'attention du géographe souhaitant retracer la morphologie de ces quartiers. Immeubles délabrés, appartements en pleine décadence, alternance entre des espaces intérieurs labyrinthiques, dégradés et réaménagés en fonction de la vente de stupéfiants, et de vastes étendues urbaines qui séparent les îlots. Les chroniques ne cessent d'ailleurs de témoigner que le quartier de Scampia est encore aujourd'hui le plus grand lieu de vente de drogue en Europe $^{3}$. Le portrait vise donc à décrire le fonctionnement spatial de la camorra : vedettes, vendeurs de drogues, voitures de protection, ou encore rémunération des familles des membres du clan en prison et explosion des conflits entre les clans, qui se manifestent non seulement avec la recrudescence des meurtres, mais aussi avec le délogement de familles entières (quand un membre a décidé de changer de clan).

L'autre typologie d'espace dominant est celle du périurbain semi-rural qui sépare les villes de Naples et Caserta. Cet espace, que les latins appelaient Campania Felix en raison de l'agriculture prolifique et de sa nature verdoyante, est devenu aujourd'hui la Terra dei Fuochi ${ }^{4}$, pour indiquer la néfaste activité qui y prend place : la décharge illégale de déchets toxiques provenant de l'Italie du nord et de l'Europe. Le paysage est composé de vieilles caves, de complexes industriels abandonnés et de terrains agricoles que les propriétaires endettés ont confié à la criminalité pour la décharge. 


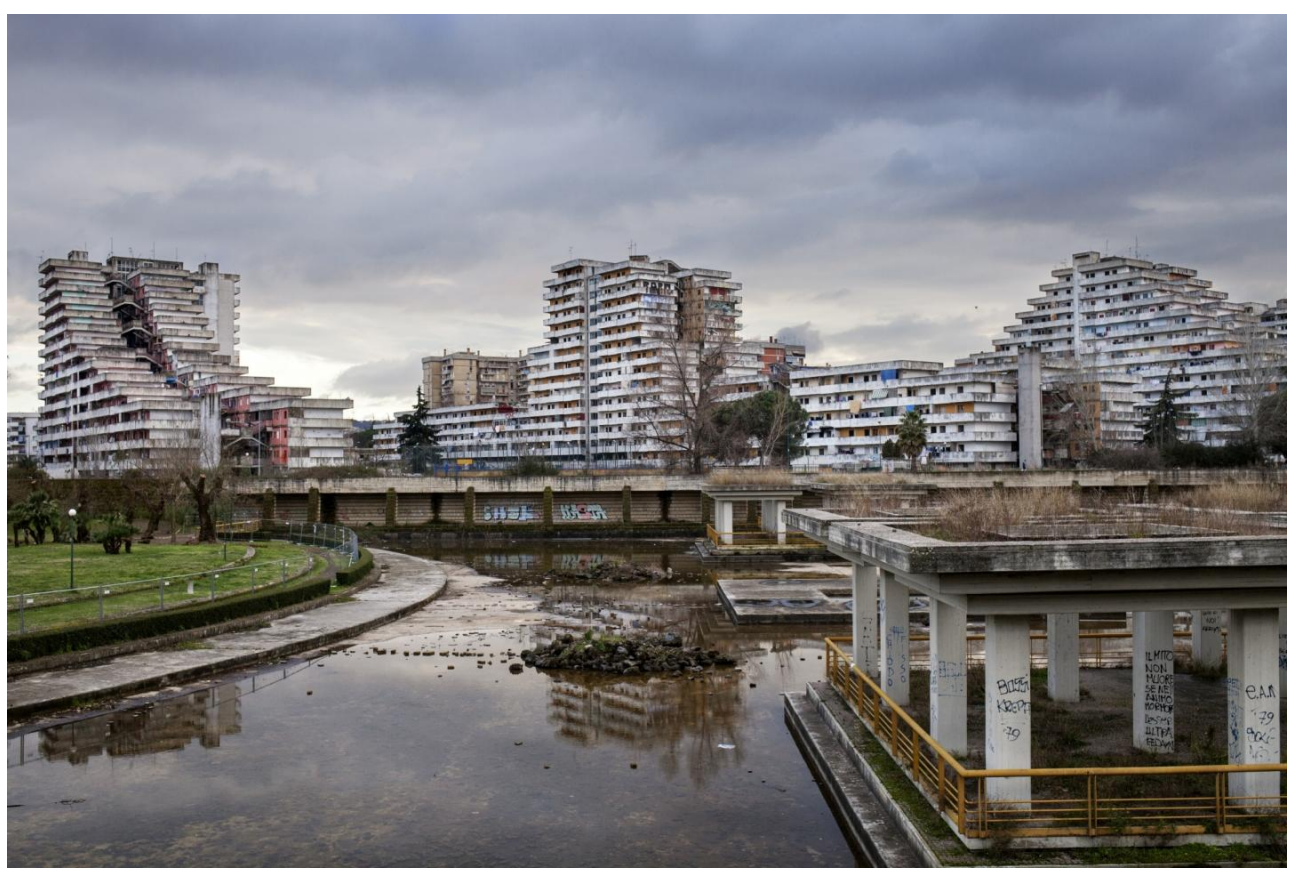

Fig. 1 : Panorama de la cité des Voiles à Scampia

19 En ce sens le film atteint pleinement son but, notamment en offrant une visibilité à des phénomènes connus mais qui n'avaient pas encore reçu l'attention qu'ils méritaient. Un autre aspect est à remarquer. Garrone, tout en gardant son style néoréaliste, parvient à inscrire son œuvre dans la longue tradition des films sur les mafias, avec un jeu d'analogies et de différences. La camorra est un phénomène criminel hétérodoxe par rapport aux autres organisations historiques (comme par exemple la Cosa Nostra sicilienne). Cette distinction se veut aussi culturelle et trouve sa visibilité dans la séquence magistrale qui ouvre le film. Dans un salon de beauté, un groupe de camorristes est en train de profiter de séances de bronzage, de soins des ongles, etc. Ils plaisantent sur les corps des uns et des autres, portent des vêtements à la mode... mais, tout à coup, ceux qui semblaient être des amis se transforment en bourreaux. Le sang coule.

20 La séquence n'est rien d'autre que la reformulation d'un des clichés les plus prolifiques de la cinématographie "mafieuse » : le barbier, un lieu masculin par excellence, lieu de décision et de discussion - mais aussi souvent de meurtre. Il suffit de penser à des classiques comme Scarface de Howard Hawks (1932), ou The Untouchables de De Palma (1987) qui montre Al Capone en train de se faire raser, ou encore The Godfather (Francis Ford Coppola, 1972) et le récent Eastern Promises (David Cronenberg, 2007). Au barbier, lieu mythique de la culture mafieuse, est substitué le salon de beauté, clair indice d'une sorte de modernisation culturelle, d'un changement de valeur qui s'éloigne des clichés. Soin du corps, bronzage et vêtements à la mode expriment de nouveaux horizons issus d'un certain mainstream massifié. Le choix de la musique est en cela exemplaire. Trois morceaux interviennent, tous appartenant au genre du "neomelodico", une évolution de la musique traditionnelle napolitaine très répandue auprès des classes populaires de Naples et plus généralement du sud de l'Italie. Les chansons sont souvent en patois, tandis que les thèmes traités alternent des histoires d'amour très romancées, souvent naïves et mielleuses, et d'autres issues du milieu criminel. C'est un élément majeur de la séquence, notamment en raison de l'importance que la musique a toujours eu dans la culture populaire napolitaine ${ }^{5}$. Toutefois, ce genre, depuis quelques années, est devenu une sorte 
de moyen d'expression de la culture camorriste. Les chanteurs ne sont pas rares qui se sont exhibés dans des cérémonies privées organisées par des membres de clans.

Le dernier de ces morceaux est le seul qui passe, à la fin de la séquence, au statut extradiégétique, faisant ainsi correspondre le refrain de la chanson avec l'apparition du titre "Gomorra ". "Notre histoire semble copiée d'un dessin animé » récite la mielleuse chanson La nostra storia de l'artiste Raffaello. Le spectateur se trouve donc face à une sorte d'oxymore audiovisuel. D'un côté : un lieu particulier, un salon de beauté, des chansons d'amour, un univers qui semble appartenir à une jetset à la mode. De l'autre : les armes, le sang, la mort.

La séquence prouve toute son efficacité dans sa capacité à montrer, en un temps relativement limité, la mutation culturelle du phénomène camorriste, tout en l'inscrivant au sein d'une longue tradition cinématographique. Ce qui persiste est malheureusement le meurtre, nous rappelant que la rénovation des formes ne change pas les contenus.

\section{Gomorra. La série.}

Quelques années plus tard, l'univers de la camorra fera l'objet d'une série télévisée dont le succès, dans le panorama italien, a été sans précédent ${ }^{6}:$ Gomorra, la série. Contrairement au film, qui s'inspire de manière directe de l'enquête de Saviano, on entre ici de plainpied dans un univers complètement fictionnel, bien que possédant une vraisemblance non négligeable. La série raconte l'histoire du clan des Savastano (nom inventé) et de ses activités criminelles liées notamment au commerce de la drogue, au racket et au contrôle sur les enchères publiques. Si les acteurs cette fois sont pour la plupart professionnels et si le patois napolitain est légèrement plus épuré pour rendre plus facile la compréhension des dialogues, les lieux de tournage sont encore marqués par un souci de réalisme. Reviennent donc les cités de la banlieue napolitaine avec, bien évidemment, les Voiles, véritable icône de la géographie camorriste. Mais l'univers diégétique s'élargit à des réalités profondément différentes comme Milan, Barcelone et Menton, pour marquer la ramification internationale de l'organisation criminelle.

Une autre différence importante concerne les choix esthétiques, notamment vis-à-vis du traitement de l'espace. L'œuvre de Garrone, on l'a vu, s'inscrit au sein d'un style visiblement néoréaliste, en essayant d'offrir un portrait d'une réalité socio-spatiale le plus neutre possible. La série, au contraire, offre des langages et des styles multiples, de manière cohérente avec un contrat fictionnel qui ne cesse jamais de faire des clins d'œil, tant à l'actualité qu'à une tradition cinématographique hétérogène. D'un côté, les extérieurs de la banlieue napolitaine respectent le souci d'adhérence et de l'autre on assiste à un usage du paysage qui suggère plutôt une posture axée sur l'imaginaire de l'espace. Comme dans le film, les monuments de Naples, son riche et merveilleux centre historique, son cityscape dominé par le Vésuve, ne sont jamais montrés. Les espaces dominants sont constitués par les vastes banlieues, les tours, les quartiers des immigrés, les petites villes du périurbain et enfin des espaces délabrés ou abandonnés. Pourtant, la série ajoute à ce panorama une imagerie inédite : des autoroutes trafiquées, des gratteciels, des portraits nocturnes de la ville et une série de séquences « urbaines » ponctuées par des thèmes musicaux récurrents.

Il s'agit d'un élément nouveau qui vise à proposer un paysage hétérogène dont les caractères rappellent les traits urbains issus des films noirs (on peut penser aux chefs 
d'œuvre de Michael Mann Heat et Collateral, 1994, 2008), de certains action movies ou encore de séries américaines comme The Wire ou The Sopranos.

L'autre différence importante entre ces deux œuvres est l'utilisation qui y est faite de la musique. Le film de Garrone limitait les insertions musicales au seul domaine diégétique. La série au contraire propose une alternance entre un registre diégétique axé sur le respect culturel du domaine de la banlieue napolitaine (encore une fois le neomelodico, mais aussi certains morceaux de rap en patois napolitain), et un autre extra-diégétique constitué par des morceaux de musique électronique composés par le groupe Mokadelic (dont le thème principal de la série). Ce n'est pas un hasard si la plupart de ces morceaux interviennent au sein de séquences descriptives comprenant des plans d'ensemble ou des travellings sur les paysages urbains.

Le résultat est un espace composite, qui alterne des traits réalistes typiques des réalités les plus dégradées du sud de l'Italie, avec d'autres qui rendent l'univers spatial de la série véritablement métropolitain, en liaison avec une imagerie urbaine cristallisée au travers du cinéma et de la télévision.

Nombreuses sont les séquences méritant l'attention du géographe. La première qui sera analysée ici sert d'introduction à la série. Ciro di Marzio et Attilio se préparent à tenter d'intimider un chef rival, Salvatore Conte. Il fait nuit et les deux hommes parcourent les routes napolitaines en voiture. Après avoir traversé un vieux site industriel, ils prennent l'autoroute. La musique est diégétique : c'est un morceau de rap en patois napolitain, apprécié par le jeune Ciro mais moins par Attilio qui avoue être «trop vieux » pour ce genre de musique. La séquence s'achève sur un plan de la voiture prenant un échangeur autoroutier. Sur le fond apparait la skyline du quartier directionnel de Naples, avec ses tours éclairées. Il s'agit d'un leitmotiv récurrent dans la série, qui vise à ajouter un véritable caractère métropolitain à la ville parthénopéenne.

Une autre séquence intéressante est celle qui présente Donna Imma Savastano (Maria Pia Calzone) à la fin du septième épisode. Son mari est en prison, son fils en Amérique du sud et elle se trouve ainsi à la tête du clan. Après une réunion avec les autres membres de l'organisation, elle se fait déposer par son chauffeur au milieu du quartier de Scampia. Elle se promène avec son chien pour s'arrêter enfin devant le paysage de la cité des Voiles. Le thème musical du groupe Mokadelic sert d'illustration sonore à la scène. Bien que la séquence soit courte, l'effet est bouleversant. La femme du boss, cadrée de dos comme dans un tableau de Friedrich, est désormais le chef de l'organisation. Elle vient de démontrer qu'elle est parfaitement capable non seulement de tenir le pouvoir, mais aussi de mettre fin aux différentes disputes pour le contrôle du trafic de drogue. Elle sourit à peine et contemple le royaume dont elle est désormais la reine. En revanche, devant elle ne se livre pas le paysage d'une nature sublime, mais au contraire la sublimation d'un cauchemar artificiel dont l'actualité dépasse malheureusement la fiction. 


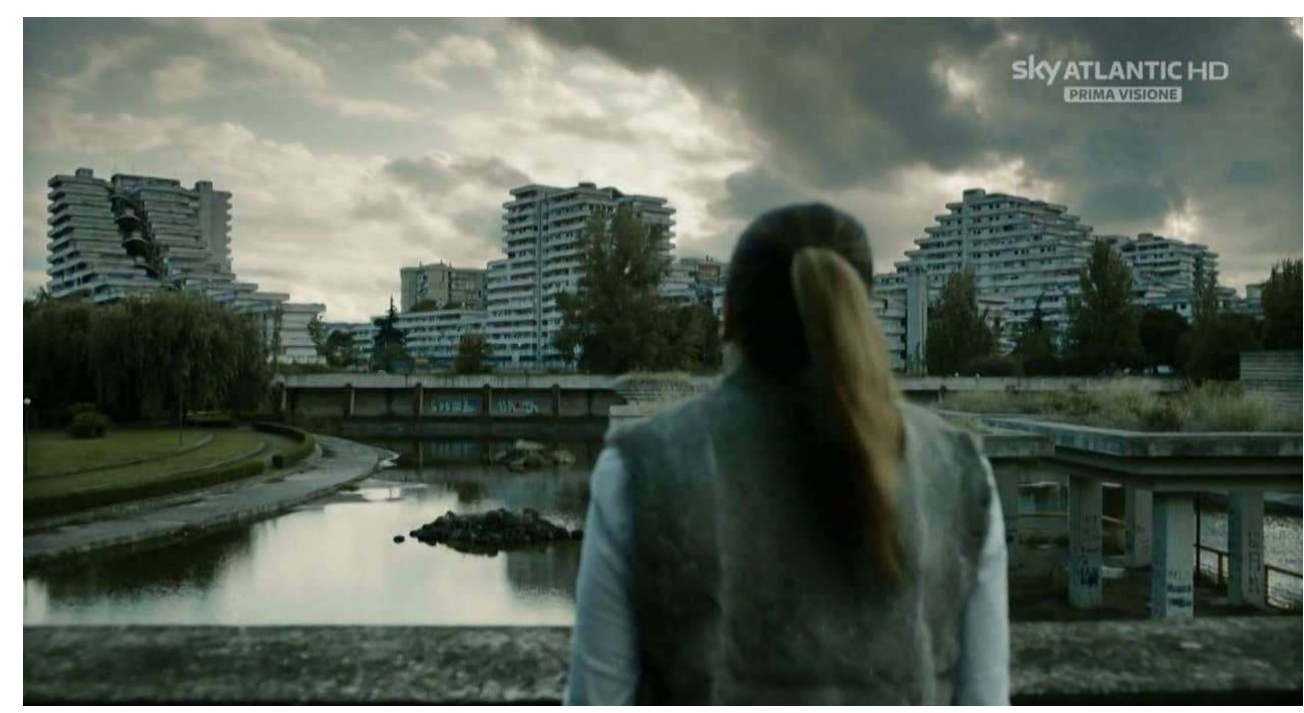

Fig. 2 : Donna Imma Savastano face à la cité des Voiles à Scampia. Gomorra la série, saison 1, ep. 7

\section{Conclusion}

L'imaginaire napolitain et son identité visuelle, construite à partir d'une longue tradition cinématographique, semblent aujourd'hui écrasés par la puissance de la culture de masse qui a porté toute son attention sur l'horreur des mafias. Qu'en est-il alors des monuments, du Vésuve ou du magnifique golfe, véritable icône parthénopéenne? Le drame de la camorra tend à les faire passer au second plan ${ }^{7}$. Cela en raison notamment des liaisons entre fiction et actualité qui sont un élément-clé du succès du genre. En ce sens, les critiques n'ont pas tardé. «À Naples, il y en a d'autres : ces imaginaires nuisent à la réputation de la ville ", peut-on synthétiser. On se trouve alors confronté au banal souci infondé, de surcroît - de l'influence négative de ces produits médiatiques. Ils auraient pour effet pervers serait celui de glorifier le crime organisé, de construire un mythe autour de cette catastrophe. C'est un point de vue bien trop étroit. Tout produit proposant une visibilité à certains phénomènes s'expose à des critiques de ce genre. Plutôt que d'alimenter un débat stérile, nous préférons souligner l'importance du regard, lucide et réaliste, que le film et la série offrent sur un phénomène dont la portée dramatique est indéniable. De plus, ces choix esthétiques et formels mis en place étaient subjectifs mais non pas mensongers, et n'ont pas manqué pour autant de représenter l'identité napolitaine. Ils l'ont enrichie, au contraire, en permettant une meilleure compréhension d'une réalité socio-spatiale qui est dans son ontologie-même variée et hétérogène, composée de beautés mais aussi de profondes laideurs. Ce n'est pas au cinéma ou à la télévision de changer le monde. Cependant, avec prudence et lucidité analytique, les produits culturels de masse tels que les films ou les séries peuvent apporter leur contribution non négligeable à une meilleure compréhension du réel, notamment dans ses aspects les plus néfastes et problématiques.

\section{Bibliographie}

Bazin, André. Qu'est-ce que le cinéma. Paris : Cerf, 1976. 
Foucher, Michel. “Du désert, paysage du western", in Hérodote $\mathrm{n}^{\circ} 7$ troisième trimestre 1977. pp. 130-147.

Gardies, André. L'espace au cinéma. Paris : Méridiens-Klincksieck, 1993.

Godelier, Maurice. L'idéel et le matériel : pensées, économies, sociétés. Paris : Fayard, 1984.

Lussault, Michel. L'homme spatial : La construction sociale de l'espace humain. Paris : Seuil, 2007.

Saviano, Roberto. Gomorra. Milan : Arnoldo Mondadori Editore, 2006. Ed. Française, Saviano

Roberto et Vincent Raynaud (trad.). Gomorra : dans l'empire de la camorra. Paris : Folio, 2006.

Scheibling, Jacques. Qu'est-ce que la géographie. Paris : Hachette, 2011 (IIème édition).

Schifano, Laurence. Le cinéma italien de 1945 à nos jours : crise et création. Paris : Nathan, 1995.

Scialo, Pasquale. La Canzone Napoletana. Roma : Newton \& Compton, 1998.

Staszak Jean-François (coord.). Annales de géographie. n 695-696 Géographie et cinéma, JanvierAvril 2014.

\section{Internet}

Basso, Fabrizio. « Il ritorno di Gomorra, tra nuove alleanze e donne criminali », Sky Atlantic, 09.05.2016. Repéré à http://skyatlantic.sky.it/skyatlantic/news/2016/05/09/gomorra-s2-lancioconferenza-stampa.html, consulté le 18 septembre 2016.

Pollichieni, Luciano. " Come Scampia è diventata Scampia e come può risorgere », Limes, 04.05.2016. Repéré à http://www.limesonline.com/come-scampia-e-diventata-scampia-e-comepuo-risorgere/91259, consulté le 17 juillet 2016.

Procaccini, Di Enrica. « Sgarbi attacca Saviano: 'Con Gomorra ha danneggiato l'immagine di Napoli...' ", Il Sud, 26.03.2015. Repéré à http://www.ilsudonline.it/sgarbi-attacca-saviano-congomorra-ha-danneggiato-limmagine-di-napoli/, consulté le 18 septembre 2016.

Ruotolo, Guido. « Cento milioni di euro l'anno il business che fa sparare i clan. », La Stampa Italia, 10.09.2012. Repéré à http://www.lastampa.it/2012/09/10/italia/cronache/cento-milioni-di-eurol-anno-il-business-che-fa-sparare-i-clan-dFP1k7776nvhX9q8hZu4OK/pagina.html, consulté le 19 juin 2016.

Saviano, Roberto. « Canzone criminale, la musica di Gomorra », La Republica, 12.02.2012. Repéré à http://www.repubblica.it/spettacoli-e-cultura/2012/02/12/news/

saviano_neomelodici-29737271/, consulté le 17 juillet 2016.

« Cannavaro inciampa su Napoli 'Gomorra da Oscar ma non giova a Italia' », La Republica, 06.01.2009. Repéré à http://www.repubblica.it/2009/01/sezioni/cronaca/cannavaro-gomorra/ cannavaro-gomorra/cannavaro-gomorra.html, consulté le 17 juin 2016.

«Sky rinova Gomorra la serie per una seconda stagione », Coming Soon, n.d. Repéré à http:// www.comingsoon.it/serietv/news/sky-rinnova-gomorra-la-serie-per-una-seconda-stagione/ n33619/, consulté le 18 septembre 2016.

Filmographie

Collateral, Michael Mann, 2004.

Eastern Promises, David Cronenberg, 2007. 
Godfather (The), Francis Ford Coppola, 2010.

Gomorra, Matteo Garrone, 2008.

Heat, Michael Mann, 1999.

Scarface, Howard Hawks, 1932.

Untouchables (The), Brian de Palma, 1987.

\section{Sériographie}

Gomorra la serie, SKY, 2014-2016.

Sopranos (The), HBO, 1999-2007.

Wire (The), HBO, 2002-2008.

\section{NOTES}

1. Le statut épistémologique de la géographie est considéré comme fluide. La cartographie, c'està-dire les études portant sur les représentations de l'espace, ont depuis toujours été au cœur de la discipline. En revanche les approches se multiplient, rendant difficile le repérage d'une identité épistémologique stable. À ce propos nous conseillons les essais de Jacques Scheibling (2011) et de Michel Lussault (2007).

2. Cinema civil : «Les années 1960-1975 voient naître en Italie des films qui ont pour but de témoigner et d'enquêter sur la réalité et le pouvoir politique » Laurence Schifano (1995).

3. À partir du travail de Saviano, qui a permis de mettre en lumière le phénomène de la Camorra, les enquêtes journalistiques se sont multipliées de façon exponentielle. L'article signé par Guido Ruotolo sur La Stampa (2012) en est un exemple, ou encore le dossier de Luciano Pollichieni publié par la revue italienne de géopolitique Limes (2016)

4. L'expression Terra dei Fuochi («terres des feux ») fut utilisée pour la première fois en 2003 au sein du rapport rédigé par l'association de sauvegarde environnementale Legamabiente concernant les liens entre le crime organisé et les délits liés à l'environnement.

5. L'ouvrage de Pasquale Scialo La Canzone Napoletana (1998) est une référence en la matière. Pour l'évolution contemporaine du genre «neomelodico», voir l'article de Roberto Saviano paru sur le quotidien La Repubblica (2012)

6. Selon le site officiel de Sky Atlantic (2016), en 2014 la série avait déjà été vendue à plus de cinquante pays dans le monde. En 2016, en pleine diffusion de la deuxième saison, le site annonce qu'elle est cette fois vendue à 170 pays. À ce propos voir aussi l'annonce faire sur le site Coming Soon (n.d.).

7. De nombreux critiques craignaient que ce type de représentations nuise d'une façon ou d'une autre à l'image de Naples. Ces critiques se sont pour la plupart exprimées sur des blogs, comme par exemple celui du quotidien napolitain Il Mattino, ou encore dans de nombreuses enquêtes télévisées réalisées dans les banlieues de la ville parthénopéenne. Quelques célébrités s'expriment sur le sujet, comme le critique d'art Vittorio Sgarbi dans une interview donnée à Di Enrica Procaccini au journal Il Sud (2015) ou encore le footballeur italien et capitaine de l'équipe 
d'Italie qui fut championne du monde en 2006 : Fabio Cannavaro, dont l'interview est parue sur le quotidien La Repubblica (2009).

\section{AUTEUR}

\section{ALFONSO PINTO}

Alfonso Pinto est un géographe italien. Il travaille depuis 2012 sur une thèse consacrée aux imaginaires urbains de la catastrophe au cinéma à l'ENS de Lyon, sous la direction de Michel Lussault et Thierry Paquot. Ses axes de recherche concernent plus généralement les rapports entre cinéma et géographie et les régimes de visibilité urbains. 